\title{
Nível de transparência por meio da evidenciação de informações obrigatórias de municípios do Rio Grande do
}

\begin{abstract}
Vagner Naysinger Machado
Mestrado em andamento em Ciências Contábeis pela Universidade do Vale do Rio dos

Sinos - UNISINOS

Rua Lima e Silva, 331. Apto 601. Centro. Porto Alegre/RS. CEP: 90050-101

E-mail: vagner29colorado@gmail.com
\end{abstract}

Simone Beatriz Santos da Silva Marques

Mestrado em andamento em Ciências Contábeis pela Universidade do Vale do Rio dos

Sinos - UNISINOS

Rua Frederico Carlos Gomes, 35. Vila Nova. Porto Alegre/RS. CEP: 91750-050

E-mail:si_beatriz@hotmail.com

Clea Beatriz Macagnan

Doutorado em Criação, Estratégia e Gestão de Empresas pela Universidade Autônoma

de Barcelona

Coordenadora do Programa de Pós-graduação em Ciências Contábeis da Universidade

do Vale do Rio dos Sinos - UNISINOS

Avenida Unisinos, 950. Bairro Cristo Rei. São Leopoldo/RS. CEP 93022-000

E-mail: clea@unisinos.br

\section{RESUMO}

O presente estudo teve como objetivo mensurar o nível de transparência, por meio da evidenciação de informações obrigatórias nas páginas eletrônicas de quarenta e um municípios do Rio Grande do Sul, Brasil, com população superior a 50 mil habitantes. 0 nível de transparência foi medido a partir de trinta e dois indicadores representativos de um conjunto de informações explicitadas na Lei de Responsabilidade Fiscal (LRF) e na Lei de Acesso à Informação (LAI). Para cada indicador evidenciado foi atribuído o valor 1 , e quando não evidenciado o valor 0 , o que possibilitou obter o percentual de indicadores evidenciados por município, mensurando o nível de transparência dos mesmos. Os resultados apontam baixo nível de transparência das informações requeridas nas leis objeto de estudo por parte dos municípios da amostra e a subutilização da Internet por parte das prefeituras municipais.

Palavras-chave: Transparência. Evidenciação. Gestão Pública.

Level of transparency through required disclosure of information of municipalities of Rio Grande do Sul - Brasil 
Nível de transparência por meio da evidenciação de informações obrigatórias de municípios do

Rio Grande do Sul

Vagner Naysinger Machado, Simone Beatriz Santos da Silva Marques, Clea Beatriz Macagnan

\section{ABSTRACT}

This study aimed to measure the level of transparency through the disclosure of required information in web pages of 41 municipalities in Rio Grande do Sul, Brazil, with population exceeding 50,000 inhabitants. The level of transparency was measured from thirty-two indicators representing a set of explicit information in the Fiscal Responsibility Law (LRF) and the Law on Access to Information (LAI). For each indicator was assigned a value 1 , and when not evidenced the value 0 , which allowed to obtain the percentage of indicators evidenced by municipality, measuring the level of transparency of theme. The results indicate a low level of transparency of information required by law object of study by the municipalities of the sample and the under-utilization of the Internet by the municipalities.

Keywords: Transparency. Disclosure. Public Management.

\section{INTRODUÇÃO}

A Constituição Federal de 1988 instituiu o Estado Democrático de Direito no Brasil, como modelo de funcionamento da sociedade. Uma das condições de existência de uma democracia é o controle social por meio da participação popular nas ações da Administração Pública, sendo que o nível e qualidade da transparência na gestão pública é um fator determinante para exercício do controle social por parte da sociedade (ARRUDA; TELES, 2010). A transparência das contas públicas relaciona-se diretamente com a evidenciação das informações à sociedade, e neste sentido a evidenciação contábil constitui-se em um meio pelo qual o gestor público pode demonstrar à sociedade que seus atos são compatíveis com os valores e regras estabelecidas (AVELINO; COLAUTO; CUNHA, 2010). As práticas de transparência na administração pública devem ir além da simples divulgação de informações alcançando um nível de evidenciação capaz de possibilitar à sociedade julgar as ações dos seus gestores (SACRAMENTO; PINHO, 2007).

A legislação brasileira estabelece um conjunto de dispositivos que normatizam os aspectos de caráter obrigatório quanto à evidenciação de informações por parte dos órgãos públicos. Nesse contexto tem-se a Lei Complementar n 101 de 04 de maio de 2000, conhecida como Lei de Responsabilidade Fiscal (LRF), integrando o processo de 
Nível de transparência por meio da evidenciação de informações obrigatórias de municípios do

Rio Grande do Sul

Vagner Naysinger Machado, Simone Beatriz Santos da Silva Marques, Clea Beatriz Macagnan

reforma do Estado como instrumento de gestão da administração pública gerencial (SACRAMENTO; PINHO, 2007). Outro dispositivo legal que merece destaque é a Lei no 12.527 de 18 de novembro de 2011, que regula o acesso a informações previsto na Constituição Federal, conhecido como Lei de Acesso à Informação (LAI). Tanto a LRF como a LAI consideram a divulgação das informações em páginas eletrônicas um meio de evidenciação de amplo acesso por parte da sociedade (LOCK, 2003; SANTANA JUNIOR, 2008).

A República Federativa do Brasil é formada pela união dos entes federados (União, Estados-Membros, Distrito Federal e Municípios). Dentre os entes públicos, os municípios são os mais próximos da sociedade, de tal forma que a gestão pública municipal pode ser considerada como a principal responsável pela prestação dos serviços (GERIGK, 2008). Neste cenário, surgiu a questão problema central da pesquisa: Qual é o nível de transparência das informações obrigatórias evidenciadas por municípios do Rio Grande do Sul com população superior a 50 mil habitantes?

Portanto, o objetivo do estudo foi mensurar o nível de transparência de informações obrigatórias evidenciadas pela administração pública dos municípios do Rio Grande do Sul com mais de 50 mil habitantes em suas respectivas páginas eletrônicas disponíveis na Internet, fazendo com que possa ser utilizado como ferramenta para auxiliar a sociedade no efetivo controle social, pois ao mensurar o nível de transparência das prefeituras nas suas páginas eletrônicas, dá-se destaque a política de evidenciação das informações dos municípios com objetivo de transparência das contas públicas municipais.

O artigo inicia com esta introdução, seguida da revisão de literatura sobre o tema transparência e estudos anteriores quanto à transparência na gestão pública municipal. A seguir é apresentada a metodologia utilizada no estudo. Logo após são apresentados e analisados os achados obtidos com a pesquisa, seguidos das considerações finais do estudo e das referências utilizadas no seu desenvolvimento. 
Nível de transparência por meio da evidenciação de informações obrigatórias de municípios do

Rio Grande do Sul

Vagner Naysinger Machado, Simone Beatriz Santos da Silva Marques, Clea Beatriz Macagnan

\section{REVISÃO DA LITERATURA}

Nessa seção apresenta-se a revisão da literatura teórica, abordando os temas: Governança Pública, Transparência por meio da Evidenciação Obrigatória, Lei de Responsabilidade Fiscal e Lei de Acesso à Informação e estudos anteriores quanto à transparência municipal.

\subsection{Governança Pública}

O Estado como instrumento de organização política de um povo, deve ser entendido como um sistema de funções que doutrinam e coordenam os meios para atingir determinados objetivos e como um conjunto de órgãos que tenham como propósito exercer essas funções (SILVA, 2009). Nesse sentido, a função da gestão pública é disponibilizar os serviços públicos necessários à população (GERIGK, 2008).

A partir da década de 1990 novos conceitos quanto à gestão dos recursos públicos foram adotados pela maioria dos países ocidentais (KRISTIANSEN et al. 2008), fazendo com que na modernização da administração pública ganhe destaque os princípios de governança (FERREIRA, 1996). Esta se refere à aquisição e distribuição de poder na sociedade, e para o desenvolvimento de uma boa governança deve-se considerar um grupo de princípios, práticas ou elementos principais (BHATTA, 2003). Dentre os princípios de boa governança, destaca-se a transparência na evidenciação das informações.

No Brasil a promulgação da LRF, em 2000, está inserida no processo de mudança da gestão pública brasileira. A LRF é o marco inicial da gestão fiscal responsável na administração pública do Brasil (TOLENTINO et al., 2002). Mais recentemente, em 2011, a adoção da LAI reforça os aspectos relacionados à busca de uma gestão pública mais gerencial, com um enfoque na transparência mediante a evidenciação das informações quanto as ações públicas em favor da sociedade. 
Nível de transparência por meio da evidenciação de informações obrigatórias de municípios do

Rio Grande do Sul

Vagner Naysinger Machado, Simone Beatriz Santos da Silva Marques, Clea Beatriz Macagnan

\subsection{Transparência}

Dentre as ações consideradas como boas práticas de governança se destacam aquelas que visam aprimorar os mecanismos de transparência. A necessidade de transparência é verificada em diversas áreas como direitos humanos, política monetária e de segurança, bem como no meio ambiente (GUPTA, 2008). Segundo Relly e Sabharwal (2009) nas últimas décadas há uma tendência mundial quanto a uma reforma nas políticas de transparência com foco na evidenciação e acesso às informações públicas. A incapacidade da sociedade em avaliar as ações dos gestores públicos, somada ao envolvimento destes em casos de corrupção, pode ser considerada um dos fatores que explicam este movimento no sentido de uma gestão pública mais transparente (FOX, 2007).

A transparência no setor público pode ser relacionada aos princípios constitucionais da legalidade e publicidade. Em países que defendem o processo democrático de acesso à informação sobre as ações dos gestores públicos, a concepção de transparência no setor público tem sido discutida de forma recorrente (SILVA, 2009), o que denota a relevância da transparência na evidenciação de informações, principalmente no que diz respeito ao conhecimento para a tomada de decisão do cidadão com relação a escolha dos seus gestores públicos. Isso porque no setor público uma maior e melhor evidenciação das informações à sociedade tende a proporcionar ações ou atos mais transparentes dos gestores públicos, facilitando o controle social da gestão pública. No próximo item, aborda-se a evidenciação de caráter obrigatório, foco deste estudo.

\subsection{Evidenciação Obrigatória}

A evidenciação das informações pode ser considerada como uma das mais importantes ferramentas para o desenvolvimento de uma política de transparência na gestão pública. Nesta esteira, Bushman, Piotroski e Smith (2004) consideram que a evidenciação está relacionada ao conceito de transparência que pode ser definido 
Nível de transparência por meio da evidenciação de informações obrigatórias de municípios do

Rio Grande do Sul

Vagner Naysinger Machado, Simone Beatriz Santos da Silva Marques, Clea Beatriz Macagnan

como a revelação de informações confiáveis e relevantes acerca da gestão das organizações.

As entidades públicas apresentam diversos aspectos que as diferem das privadas. Essas diferenças dizem respeito à evidenciação de informações obrigatórias. As evidenciações compulsórias para o setor público brasileiro estão dispostas em diversas leis, entre elas pode-se destacar a Lei ํo. 4.320/64, a Lei ํo. 9.755/98, e a Lei no. 10.028/00. No que tange a transparência da gestão pública com foco na evidenciação das informações, destaca-se a Lei Complementar № 101 de 04 de maio de 2000 - Lei de Responsabilidade Fiscal e a recente adoção da Lei oㅜ 12.527 de 18 de novembro de 2011 que regula o acesso à informação.

\subsubsection{Lei de Responsabilidade Fiscal (LRF) e Lei de Acesso à Informação (LAl)}

No Brasil, na última década, uma das mudanças mais profundas na gestão pública é a adoção da responsabilidade fiscal como princípio norteador da Administração Pública. Conforme Gerigk (2008) devido aos constantes desequilíbrios das contas públicas e das administrações irresponsáveis frente aos entes públicos, notou-se a necessidade de enrijecer o controle sobre as finanças públicas e fortalecer os instrumentos de transparência e planejamento. Nesse contexto foi promulgada a Lei Complementar no 101, de 04 de maio de 2000 (LRF), que estabelece normas de finanças públicas voltadas para a responsabilidade na gestão fiscal e dá outras providências. A Lei inova em relação às tentativas anteriores quanto à limitação dos gastos públicos, pois trouxe o aspecto do planejamento governamental via orçamento, e a punição (responsabilidade) aos maus gestores públicos com base na transparência das contas públicas (COSTA, 2008).

O principal objetivo da LRF é a busca de uma gestão fiscal responsável e de qualidade, o que pressupõe ações planejadas e transparentes e uma simetria das contas públicas. Um dos pilares para uma gestão fiscal responsável é a transparência das ações governamentais. Esta se refere à responsabilidade dos agentes públicos darem acesso às informações aos cidadãos quanto as suas atuações, em termos de 
Nível de transparência por meio da evidenciação de informações obrigatórias de municípios do

Rio Grande do Sul

Vagner Naysinger Machado, Simone Beatriz Santos da Silva Marques, Clea Beatriz Macagnan

gestão dos recursos públicos. Assim, a transparência da gestão dos recursos públicos é uma condição sine qua non (SACRAMENTO; PINTO, 2007).

$O$ art. 48 da LRF elenca os instrumentos de transparência na gestão fiscal tais como: os planos, orçamentos e leis de diretrizes orçamentárias; as prestações de contas e o respectivo parecer prévio; o Relatório Resumido da Execução Orçamentária e o Relatório de Gestão Fiscal. Com isso, a LRF pretende ampliar a transparência dos atos de planejamento e controle para cada período. O parágrafo único do artigo $48 \mathrm{da}$ LRF acrescenta como formas de assegurar a transparência, a divulgação em tempo real das informações quanto à execução orçamentária e financeira e o incentivo a participação popular e a realização de audiências públicas quando da fase de elaboração e discussão dos planos, leis de diretrizes orçamentárias e orçamentos. Já o artigo 9 da LRF faz menção ao calendário das audiências públicas referentes a questões orçamentárias, e quanto as avaliações das metas fiscais. A LRF propugna a ampla divulgação destes instrumentos de transparência, inclusive em meios eletrônicos de acesso público.

Já a promulgação da Lei oㅜ 12.527 de 18 de novembro de 2011 (LAl) tem como objetivo central a disseminação da cultura de transparência da gestão pública brasileira por meio da regulamentação do direito de acesso às informações. A institucionalização de mecanismos de acesso a informação é uma das formas encontradas pelas democracias para coibir possíveis abusos de poder e desrespeito ao direito humano fundamental de acesso às informações públicas (ANDI, 2009). A LAI normatiza que é dever do Estado garantir o acesso à informação através de procedimentos de forma transparente, clara e em linguagem de fácil compreensão, assegurando assim a gestão transparente da informação, proporcionando amplo acesso a ela e sua divulgação.

Com a edição da LAI o Brasil dá um importante passo em sua trajetória de transparência pública, estabelecendo que o acesso às informações é a regra e passa a integrar a um amplo grupo de nações que reconhece as informações públicas como um bem público, o que pode fortalecer os sistemas democráticos, resultando em ganhos para as sociedades (CGU, 2011), de tal forma que a transparência pode ser um 
Nível de transparência por meio da evidenciação de informações obrigatórias de municípios do

Rio Grande do Sul

Vagner Naysinger Machado, Simone Beatriz Santos da Silva Marques, Clea Beatriz Macagnan

elemento de transformação da administração pública no Brasil. Nesse sentido, a LAI exige uma ampla visibilidade das ações governamentais e estabelece diversas formas de disponibilização de informações à sociedade (TCE/RS, 2012).

A LAI estabelece que as entidades públicas devam observar, dentre outras diretrizes, o incentivo ao desenvolvimento da cultura de transparência da Administração Pública; o desenvolvimento do controle social, e a utilização da tecnologia da informação. Segundo este dispositivo legal, é dever dos órgãos e entidades públicas a promoção da divulgação em local de fácil acesso, das informações de interesse coletivo ou geral, por eles produzidas ou custodiadas. Para tal, a LAI torna obrigatória a divulgação destas informações em sítios oficiais da rede mundial de computadores (Internet) para os municípios com mais de 10 mil habitantes.

\subsubsection{Estudos Anteriores}

Pesquisou-se nas bases de dados CAPES, EBSCO e no site de busca na Internet Google ${ }$, a expressão "transparência na gestão pública municipal", e dentre os estudos encontrados destacam-se aqueles que abordaram a transparência da gestão pública municipal com enfoque na evidenciação obrigatória, objeto desse estudo.

O estudo de Lock (2003) teve como objetivo identificar o grau de transparência da gestão pública de prefeituras brasileiras, por meio da análise do cumprimento do artigo 48 da LRF. Os resultados apontam que a grande maioria dos municípios não divuga as informações exigidas pela legislação, deixando os cidadãos sem acesso, via Internet, aos dados econômico-financeiros referentes a gestão pública das prefeituras.

Styles e Tennyson (2007) identificaram o nível de acesso dos cidadãos aos dados financeiros de 300 municípios dos EUA disponíveis na Internet, com base em normas emitidas naquele pais. Um dos resultados do estudo aponta que uma proporção significativa dos municípios pesquisados não utiliza a Internet como meio de promover a transparência da gestão pública municipal.

O Estudo de Souza et al. (2008) avaliou a transparência das gestões públicas de 85 cidades do Estado de Minas Gerais (Brasil), com base na evidenciação das 
Nível de transparência por meio da evidenciação de informações obrigatórias de municípios do Rio Grande do Sul Vagner Naysinger Machado, Simone Beatriz Santos da Silva Marques, Clea Beatriz Macagnan

informações de carater compulsório de acordo com a LRF, por meio das suas respectivas páginas eletrônicas. Os achados indicam que nenhum município pesquisado evidenciou todas as informações requeridas pela legislação.

Cruz et al. (2012) em sua pesquisa tiveram como um dos objetivos identificar o nível de transparência das informações acerca da gestão pública municipal das cidades mais populosas do Brasil evidenciadas em seus portais eletrônicos disponíveis na Internet. Como um dos resultados, o estudo aponta que a média geral do índice de transparência municipal das cidades estudadas corresponde a apenas $46,22 \%$ do total, classificado pelos autores como um baixo nível de transparência.

Os estudos mencionados não abordaram os aspectos tratados na Lei de Acesso a Informação. Já o Tribunal de Contas do Estado do Rio Grande do Sul (TCE-RS, 2012), publicou em setembro 2012 o estudo intitulado "Os Portais dos Municípios Gaúchos (Poder Executivo) - Análise em face da Lei Federal n 12.527/2011”. Com este estudo o TCE/RS objetivou verificar o desempenho dos municípios quanto ao cumprimento da Lei de Acesso à Informação. Dentre outros resultados, o TCE/RS concluiu que apenas 20 de um total de 496 municípios apresentam indicações claras à LAl e que $95,3 \%$ das prefeituras sequer oferecem aos cidadãos meios de acesso aos dados da Administração Municipal, evidenciando que a maioria das prefeituras do estado do Rio Grande do Sul não cumpre os dispositivos da LAI.

\section{METODOLOGIA}

Neste item apresentam-se os aspectos metodológicos deste estudo. A seleção da população e da amostra, a fonte e procedimentos de coletas de evidências e as técnicas utilizadas para a análise dos dados.

A população da pesquisa compreende todos os 496 municípios do Estado do Rio Grande do Sul. Dentre estes foram escolhidos os municípios com mais de 50 mil habitantes, uma vez que a LRF permite que as prefeituras com menos de 50 mil habitantes evidenciem menor número de relatórios, em períodos inferiores aos demais. 
Nível de transparência por meio da evidenciação de informações obrigatórias de municípios do Rio Grande do Sul

Vagner Naysinger Machado, Simone Beatriz Santos da Silva Marques, Clea Beatriz Macagnan

Desta forma, com base nos dados do censo IBGE (2010), chegou-se ao número de 42 municípios pertencentes ao estado do Rio Grande do Sul com população superior a 50 mil habitantes.

Para definição da amostra foram localizadas as respectivas páginas eletrônicas dos 42 municípios, utilizando-se como ferramenta de busca a página eletrônica do Google® (http://www.google.com.br). Pesquisou-se a seguinte expressão: "prefeitura municipal de (nome da cidade)", considerando-se apenas as páginas eletrônicas oficiais das prefeituras, ou seja, aquelas com extensão "gov". Este procedimento de busca ocorreu nos primeiros 10 (dez) dias do mês de janeiro de 2013. A página eletrônica do município de São Gabriel encontrava-se fora do ar, o que impossibilitou a coleta dos dados, ficando o mesmo excluído da amostra que passou a ser formada por 41 municípios. A Tabela 1 elenca os municípios da amostra, suas respectivas populações e páginas eletrônicas oficiais. 
Nível de transparência por meio da evidenciação de informações obrigatórias de municípios do

Rio Grande do Sul

Vagner Naysinger Machado, Simone Beatriz Santos da Silva Marques, Clea Beatriz Macagnan

Tabela 1- Amostra - Listagem dos municípios, populações e páginas eletrônicas

\begin{tabular}{|c|c|c|c|}
\hline & Municípios & População & Páginas Eletrônicas \\
\hline 1 & Porto Alegre & 1.409 .939 & http://www.portoalegre.rs.gov.br \\
\hline 2 & Caxias do Sul & 435.482 & http://www.caxias.rs.gov.br \\
\hline 3 & Pelotas & 327.778 & http://www.pelotas.rs.gov.br \\
\hline 4 & Canoas & 324.025 & http://www.canoas.rs.gov.br \\
\hline 5 & Santa Maria & 261.027 & http://www.santamaria.rs.gov.br \\
\hline 6 & Gravataí & 255.762 & http://www.gravatai.rs.gov.br \\
\hline 7 & Viamão & 239.234 & http://www.viamao.rs.gov.br \\
\hline 8 & Novo Hamburgo & 239.051 & http://www.novohamburgo.rs.gov.br \\
\hline 9 & São Leopoldo & 214.210 & https://www.saoleopoldo.rs.gov.br \\
\hline 10 & Rio Grande & 197.253 & http://www.riogrande.rs.gov.br \\
\hline 11 & Alvorada & 195.718 & http://www.alvorada.rs.gov.br \\
\hline 12 & Passo Fundo & 184.869 & http://www.pmpf.rs.gov.br \\
\hline 13 & Sapucaia do Sul & 130.988 & http://www.sapucaiadosul.rs.gov.br \\
\hline 14 & Uruguaiana & 125.507 & http://www.uruguaiana.rs.gov.br \\
\hline 15 & Cachoeirinha & 118.294 & http://www.cachoeirinha.rs.gov.br \\
\hline 16 & Santa Cruz do Sul & 118.287 & http://www.santacruz.rs.gov.br \\
\hline 17 & Bagé & 116.078 & http://www.bage.rs.gov.br \\
\hline 18 & Bento Gonçalves & 107.341 & http://www.bentogoncalves.rs.gov.br \\
\hline 19 & Erechim & 96.105 & http://www.pmerechim.rs.gov.br \\
\hline 20 & Guaíba & 95.230 & http://www.guaiba.rs.gov.br \\
\hline 21 & Cachoeira do Sul & 83.827 & http://www.cachoeiradosul.rs.gov.br \\
\hline 22 & Santana do Livramento & 82.513 & http://www.santanadolivramento.rs.gov.br \\
\hline 23 & Esteio & 80.669 & http://www.esteio.rs.gov.br \\
\hline 24 & ljuí & 78.920 & http://www.ijui.rs.gov.br \\
\hline 25 & Alegrete & 77.673 & http://www.alegrete.rs.gov.br \\
\hline 26 & Santo Angelo & 76.304 & http://www.santoangelo.rs.gov.br \\
\hline 27 & Sapiranga & 75.020 & http://www.sapiranga.rs.gov.br \\
\hline 28 & Lajeado & 71.481 & http://www.lajeado.rs.gov.br \\
\hline 29 & Santa Rosa & 68.595 & http://www.santarosa.rs.gov.br \\
\hline 30 & Venâncio Aires & 65.964 & http://www.venancioaires.rs.gov.br \\
\hline 31 & Farroupilha & 63.641 & http://www.farroupilha.rs.gov.br \\
\hline 32 & Cruz Alta & 62.825 & http://www.cruzalta.rs.gov.br \\
\hline 33 & Camaquã & 62.759 & http://www.camaqua.rs.gov.br \\
\hline 34 & São Borja & 61.671 & http://www.saoborja.rs.gov.br \\
\hline 35 & Vacaria & 61.345 & http://www.vacaria.rs.gov.br \\
\hline 36 & Campo Bom & 60.081 & http://www.campobom.rs.gov.br \\
\hline 37 & Montenegro & 59.436 & https://www.montenegro.rs.gov.br \\
\hline 38 & Carazinho & 59.301 & http://www.carazinho.rs.gov.br \\
\hline 39 & Taquara & 54.656 & http://www.taquara.rs.gov.br \\
\hline 40 & Canguçu & 53.268 & http://www.cangucu.rs.gov.br \\
\hline 41 & Parobé & 51.481 & http://www.parobe.rs.gov.br \\
\hline
\end{tabular}

Fonte: Elaborado pelos Autores.

O exame das informações disponíveis nas páginas eletrônicas foi efetuado

durante o mês de janeiro de 2013. Primeiramente, buscaram-se as informações no 
Nível de transparência por meio da evidenciação de informações obrigatórias de municípios do

Rio Grande do Sul

Vagner Naysinger Machado, Simone Beatriz Santos da Silva Marques, Clea Beatriz Macagnan

mapa do site, depois foram utilizados os links disponíveis e por último tentou-se a ferramenta de busca do site. Dessa forma, para atingir o objetivo central do estudo, selecionou-se o conjunto de 32 indicadores de informações obrigatórias, 16 deles que atendiam a LRF e outros 16 indicadores que atendiam a LAI.

A seguir, apresenta-se o conjunto de indicadores de informações de caráter obrigatório utilizado neste estudo e suas bases legais:

1. Plano Plurianual (PPA) vigente - art. 48 LRF;

2. Plano Plurianual (PPA) anterior - art. 48 LRF;

3. Lei de Diretrizes Orçamentárias (LDO) vigente - art. 48 LRF;

4. Lei de Diretrizes Orçamentárias (LDO) anterior - art. 48 LRF;

5. Lei Orçamentária Anual (LOA) vigente - art. 48 LRF;

6. Lei Orçamentária Anual (LOA) anterior - art. 48 LRF;

7. Relatório Resumido de Execução Orçamentária (RREO) - art. 48 LRF;

8. Relatório de Gestão Fiscal (RGF) - art. 48 LRF;

9. Versão Simplificada RREO - art. 48 LRF;

10. Versão simplificada RGF - art. 48 LRF;

11. Relatório de Prestação de Contas - art.48 LRF;

12. Parecer Prévio da Prestação de Contas - art. 48 LRF;

13. Incentivo a participação popular e audiências públicas no processo de elaboração e discussão do PPA, LDO, LOA - art. 48 LRF;

14. Informações execução orçamentária e financeira em tempo real - art. 48 LRF;

15. Divulgação das datas das audiências públicas durante processo de elaboração e discussão do PPA, LDO, LOA - art. 9 LRF;

16. Divulgação das datas das audiências públicas quadrimestrais para avaliação das metas fiscais - art. 9 LRF;

17. Indicação clara a Lei de Acesso à Informação - art. 5 LAl;

18. Indicação de meios para solicitação de informações - art. 5 LAl;

19. Criação do Serviço de Informações ao Cidadão (SIC) - art. 9 LAl; 
Nível de transparência por meio da evidenciação de informações obrigatórias de municípios do

Rio Grande do Sul

Vagner Naysinger Machado, Simone Beatriz Santos da Silva Marques, Clea Beatriz Macagnan

20. Informações quanto as competências dos órgãos municipais - art. 8 LAl;

21. Informações quanto a estrutura organizacional - art. 8 LAl;

22. Divulgação do endereço, telefones e horário de atendimento - art. $8 \mathrm{LAl}$;

23. Informações relativas a repasses ou transferências de recursos - art.8 LAl;

24. Informações sobre despesas realizadas - art. $8 \mathrm{LAl}$;

25. Informações sobre licitações, editais e resultados dos certames - art. $8 \mathrm{LAl}$;

26. Informações sobre contratos celebrados - art. $8 \mathrm{LAl}$;

27. Acompanhamento de programas, ações, projetos e obras - art. 8 LAl;

28. Disponibilidade de respostas a "perguntas mais frequentes (FAQ)" - art. 8 LAl;

29. Disponibilidade de ferramenta de pesquisa - art. 8 LAl;

30. Permissão de gravação de relatórios eletrônicos - art. 8 LAl;

31. Atualização das informações disponibilizadas - art. 8 LAl;

32. Acesso às informações por pessoas portadoras de deficiências - art. 8 LAI.

A seleção destes indicadores de informações foi elaborada com base nos estudos anteriores, de forma que os indicadores de informações correspondentes ao artigo 48 LRF foram observados também nos estudos de Lock (2003), Souza et al. (2008) e Cruz et al. (2012). Ademais, os indicadores relativos ao artigo 9 da LRF também foram analisados no estudo de Cruz et al. (2012). Já os indicadores de informações que atendem a LAI também foram considerados no trabalho desenvolvido pelo TCE-RS (2012). Além disso, os indicadores de informações referentes à disponibilidade de ferramenta de pesquisa ( $n$ ‥ 29) e permissão para gravação de relatórios eletrônicos ( $n$ - .30 ) também foram examinados no estudo de Styles e Tennyson (2007).

A análise das páginas eletrônicas centrou-se no atendimento de cada um dos 32 indicadores de informações. Logo, com a finalidade de quantificar o nível de transparência municipal por meio de suas páginas eletrônicas, atribuiu-se 1 (um) ponto aos indicadores de informações evidenciadas nas homepages dos municípios, e 
Nível de transparência por meio da evidenciação de informações obrigatórias de municípios do

Rio Grande do Sul

Vagner Naysinger Machado, Simone Beatriz Santos da Silva Marques, Clea Beatriz Macagnan

nenhum ponto aos indicadores não evidenciados. Desta forma, o nível de transparência municipal poderia variar de 0 a 32 pontos.

Quanto aos indicadores de informações referentes ao PPA (Plano Plurianual), considerou-se "vigente" o PPA relativo ao período de 2010 a 2013, e "anterior" o plano referente ao período de 2006 a 2009. Já em relação aos indicadores de informações quanto a LDO (Lei de Diretrizes Orçamentária) e LOA (Lei Orçamentária Anual), considerou-se como "vigente" as leis relativas ao ano de 2012 e "anterior" os dispositivos relativos ao ano de 2011.

\section{APRESENTAÇÃO E ANÁLISE DAS EVIDÊNCIAS}

Como resultado da análise da evidenciação individual dos indicadores de informações compulsórias, chegou-se ao nível de transparência da gestão pública municipal. A Tabela 2 elenca os cinco municípios com maiores níveis de transparência conforme os indicadores de informações analisados em suas respectivas páginas eletrônicas.

Tabela 2 - Cinco municípios mais transparentes

\begin{tabular}{|c|c|c|c|}
\hline & Município & Pontuação & $\%$ \\
\hline & Novo Hamburgo & 24 & 75,00 \\
\hline & Passo Fundo & 23 & 71,88 \\
\hline & Canoas & 22 & 68,75 \\
\hline & Porto Alegre & 22 & 68,75 \\
\hline & São Leopoldo & 21 & 65,63 \\
\hline
\end{tabular}

Fonte: Dados da pesquisa.

Conforme observado na Tabela 2 o município com maior nível de transparência é Novo Hamburgo. O município evidencia em sua página eletrônica vinte quatro indicadores de informações, sendo 12 referentes a Lei de Responsabilidade Fiscal e 12 são explicitados pela Lei de Acesso à Informação, o que representa uma evidenciação de $75 \%$. Nota-se, assim, que nenhum município estudado divulga todos os indicadores 
Nível de transparência por meio da evidenciação de informações obrigatórias de municípios do Rio Grande do Sul Vagner Naysinger Machado, Simone Beatriz Santos da Silva Marques, Clea Beatriz Macagnan de informações analisados. Este dado corrobora com os resultados encontrados nos estudos de Lock (2003), Souza et al. (2008) e Cruz et al. (2012).

A Tabela 3 apresenta os cinco municípios com os níveis mais baixos de transparência dos indicadores examinados.

Tabela 3 - Cinco municípios menos transparentes

\begin{tabular}{|c|c|c|c|}
\hline & Município & Pontuação & $\%$ \\
\hline & Santana do Livramento & 9 & 28,13 \\
\hline & Alvorada & 8 & 25,00 \\
\hline & Cachoeira do Sul & 8 & 25,00 \\
\hline & Viamão & 8 & 25,00 \\
\hline & Sapiranga & 6 & 18,75 \\
\hline
\end{tabular}

Fonte: Dados da pesquisa.

De acordo com a Tabela 3, o município com o menor nível de transparência é Sapiranga que evidencia apenas seis indicadores. O município disponibiliza em sua página eletrônica apenas informações quanto à versão simplificada do Relatório Resumido de Execução orçamentária (RREO) e do Relatório de Gestão Fiscal (RGF), ambos exigidos pelo artigo 48 da LRF. Os outros quatro indicadores evidenciados são previstos pelo artigo 9을 da LAl e correspondem às informações quanto à estrutura organizacional, as competências dos órgãos municipais, as despesas realizadas e a permissão para gravação dos relatórios eletrônicos.

O nível médio de transparência dos municípios analisados é de 15,54 pontos, o que corresponde a $48,55 \%$ da pontuação máxima. Este resultado se assemelha aos achados do estudo de Cruz et al., (2012), pois seus resultados indicam que a média geral do índice de transparência municipal corresponde apenas a $46,22 \%$ do total, o que é considerado pelos autores um baixo índice de transparência. Como resultado da análise das informações disponíveis nas páginas eletrônicas dos 41 municípios da amostra, tem-se que uma das mais importantes ferramentas de comunicação, a Internet, é subutilizada pelos gestores públicos destas entidades, fato também constatado por Lock (2003), Styles e Tennyson (2007), Souza et al. (2008) e Cruz et al. (2012). 
Nível de transparência por meio da evidenciação de informações obrigatórias de municípios do

Rio Grande do Sul

Vagner Naysinger Machado, Simone Beatriz Santos da Silva Marques, Clea Beatriz Macagnan

Ao se analisar os indicadores de informações evidenciados nas páginas eletrônicas dos municípios e suas respectivas bases legais, verifica-se que a LRF apresenta um nível de transparência de $46,47 \%$ do total, enquanto que a LAI 53,53\%, ou seja, nos municípios estudados tende-se a evidenciar com maior frequência as informações previstas na LAI, em comparação com a LRF. Na Tabela 4 apresentam-se os indicadores de informações previstos na LRF evidenciados por até $30 \%$ dos municípios pesquisados.

Tabela 4 - Indicadores da LRF evidenciados por até 30\% dos municípios

\begin{tabular}{|c|c|c|}
\hline Indicadores (Informações) & № Municípios & $\%$ \\
\hline $\begin{array}{c}\text { Informações sobre execução orçamentária e financeira em tempo } \\
\text { real }\end{array}$ & 8 & 19,51 \\
\hline Relatório de prestação de contas & 2 & 4,88 \\
\hline Parecer prévio da prestação de contas & 2 & 4,88 \\
\hline $\begin{array}{c}\text { Divulgação das datas das audiências públicas quadrimestrais para } \\
\text { avaliação das metas fiscais }\end{array}$ & 1 & 2,43 \\
\hline $\begin{array}{c}\text { Incentivo a participação popular e audiências públicas no processo } \\
\text { de elaboração e discussão do PPA, LDO, LOA. }\end{array}$ & 0 & 0,00 \\
\hline $\begin{array}{c}\text { Divulgação das datas das audiências públicas durante processo de } \\
\text { elaboração e discussão do PPA, LDO e LOA }\end{array}$ & 0 & 0,00 \\
\hline
\end{tabular}

Fonte: Dados da pesquisa.

A Tabela 4 evidencia os indicadores de informações previstos na LRF divulgados com menor frequência pelos municípios pesquisados. Verifica-se que nenhum município evidencia indicadores referentes ao incentivo à participação popular e a divulgação das datas das audiências públicas quanto ao processo de elaboração e discussão do plano plurianual, das leis de diretrizes orçamentárias e das leis orçamentárias anuais. As datas das audiências públicas quadrimestrais referentes à avaliação das metas fiscais da gestão pública do período são divulgadas apenas pela prefeitura do município de Caxias do Sul. Já as informações quanto ao relatório de prestação de contas e seus respectivos pareceres prévios são evidenciados apenas pelos municípios de Novo Hamburgo e Alegrete, o que corresponde a apenas 4,88\% dos municípios estudados. Ademais, apenas oito prefeituras disponibilizam em suas 
Nível de transparência por meio da evidenciação de informações obrigatórias de municípios do

Rio Grande do Sul

Vagner Naysinger Machado, Simone Beatriz Santos da Silva Marques, Clea Beatriz Macagnan

páginas eletrônicas informações quanto à execução orçamentária e financeira em tempo real, como determina o artigo 48 da LRF.

A Tabela 5 elenca os indicadores de informações previstos na LRF evidenciados por $30 \%$ a $70 \%$ dos municípios pesquisados.

Tabela 5 - Indicadores da LRF evidenciados por $30 \%$ a $70 \%$ dos municípios

\begin{tabular}{|c|c|c|}
\hline Indicadores (Informações) & № Municípios & $\%$ \\
\hline Lei de Diretrizes Orçamentárias (LDO) vigente & 28 & 68,29 \\
\hline Lei Orçamentária Anual (LOA) vigente & 28 & 68,29 \\
\hline Lei Orçamentária Anual (LOA) anterior & 28 & 68,29 \\
\hline Relatório de Gestão Fiscal (RGF) & 26 & 63,41 \\
\hline Relatório Resumido de Execução Orçamentária (RREO) & 25 & 60,98 \\
\hline Plano Plurianual (PPA) anterior & 22 & 53,66 \\
\hline
\end{tabular}

Fonte: Dados da pesquisa.

Nota-se que o plano plurianual correspondente ao período de 2006 a 2009 é divulgado por $53,66 \%$ dos municípios da amostra. O relatório resumido de execução orçamentária (RREO) é evidenciado por $60,98 \%$ e o relatório de gestão fiscal (RGF) por $63,41 \%$ das prefeituras. Já a lei de diretrizes orçamentárias vigente, correspondente a LDO do ano de 2012, a lei orçamentária anual vigente (2012) e anterior (2011) são evidenciadas por vinte e oito municípios pesquisados, o equivalente a $68,29 \%$ das prefeituras analisadas.

A Tabela 6 apresenta os indicadores de informações previstos na LRF evidenciados por mais de $70 \%$ dos municípios estudados.

Tabela 6 - Informações Evidenciadas quanto a LRF por mais de $70 \%$ dos municípios

\begin{tabular}{|c|c|c|}
\hline Indicadores (Informações) & № Municípios & $\%$ \\
\hline Versão simplificada RREO & 34 & 82,93 \\
\hline Plano Plurianual (PPA) vigente & 32 & 78,05 \\
\hline Lei de Diretrizes Orçamentária (LDO) anterior & 30 & 73,17 \\
\hline Versão simplificada RGF & 30 & 73,17 \\
\hline
\end{tabular}

Fonte: Dados da pesquisa. 
Nível de transparência por meio da evidenciação de informações obrigatórias de municípios do

Rio Grande do Sul

Vagner Naysinger Machado, Simone Beatriz Santos da Silva Marques, Clea Beatriz Macagnan

A lei de diretrizes orçamentárias (LDO) correspondente ao ano de 2011 e a versão simplificada do relatório de gestão fiscal (RGF) são evidenciadas por 73,17\% das prefeituras estudadas. O plano plurianual (PPA) vigente correspondente ao período de 2009 a 2013 é divulgado por 78,05\% das prefeituras, e a versão simplificada do relatório resumido da execução orçamentária (RREO) é a informação prevista na LRF mais evidenciada, pois $82,93 \%$ dos municípios disponibilizam em suas páginas eletrônicas tal informação.

$\mathrm{Na}$ Tabela 7 constam os indicadores previstos na LAl evidenciados por até $30 \%$ dos municípios pesquisados.

Tabela 7 - Indicadores da LAI evidenciados por até 30\% dos municípios

\begin{tabular}{|c|c|c|}
\hline Indicadores (Informações) & № Município & $\%$ \\
\hline Informações relativas a repasses ou transferências de recursos & 10 & 24,39 \\
\hline Disponibilidade de respostas a "perguntas mais frequentes FAQ" & 7 & 17,07 \\
\hline Acompanhamento de programas, ações, projetos e obras & 3 & 7,32 \\
\hline Disponibilidade de acesso a informações por pessoas com deficiências & 1 & 2,44 \\
\hline
\end{tabular}

Fonte: Dados da pesquisa.

Apenas um município (Canoas) disponibiliza em sua página eletrônica acesso as informações por pessoas portadoras de deficiências. Por ter sido utilizada uma metodologia diferente dessa pesquisa, não é possível a comparação dos resultados apurados com os obtidos pelo TCE-RS (2012). Outro indicador pouco evidenciado pelos municípios pesquisados diz respeito às informações para acompanhamento de programas, ações, projetos e obras. Apenas os municípios de Canoas, Pelotas e Rio Grande $(7,32 \%)$ evidenciam em suas páginas eletrônicas tais informações. O TCE-RS (2012) apurou que $21,37 \%$ das prefeituras disponibilizavam em suas páginas eletrônicas tal informação, tendo sido considerado pelo como baixo nível de evidenciação das informações em questão.

A ferramenta disponibilidade de respostas a perguntas mais frequentes (FAQ) foi encontrada nas páginas eletrônicas de sete municípios (17,07\%), enquanto a pesquisa do TCE-RS (2012) apontou um resultado ainda pior, apenas 3,6\% das prefeituras 
Nível de transparência por meio da evidenciação de informações obrigatórias de municípios do

Rio Grande do Sul

Vagner Naysinger Machado, Simone Beatriz Santos da Silva Marques, Clea Beatriz Macagnan

disponibilizavam tal indicador. As informações quanto a repasses ou transferências de recursos são disponibilizadas nas páginas eletrônicas de 24,39\% dos municípios, percentual inferior ao obtido pelo TCE-RS (2012), o qual apurou que 30,85\% dos municípios do Rio Grande do Sul evidenciavam tais informações.

$\mathrm{Na}$ Tabela 8 constam os indicadores previstos na LAl evidenciados por $30 \%$ a $70 \%$ dos municípios pesquisados.

Tabela 8 - Informações Evidenciadas quanto a LAI entre 30\% e 70\% dos municípios

\begin{tabular}{|c|c|c|}
\hline Indicadores (Informações) & № Município & $\%$ \\
\hline Informações quanto as suas competências & 28 & 68,29 \\
\hline Disponibilidade de ferramenta de pesquisa & 23 & 56,10 \\
\hline Indicação de meios para solicitação de informações & 22 & 53,66 \\
\hline Indicação clara de acesso a Lei de Acesso à Informação (LAI) & 20 & 48,78 \\
\hline Informações sobre contratos celebrados & 15 & 36,59 \\
\hline Criação do serviço de informações ao cidadão - SIC & 14 & 34,15 \\
\hline Atualização das informações disponibilizadas & 14 & 34,15 \\
\hline
\end{tabular}

Fonte: Dados da pesquisa.

Os indicadores relacionados com a atualização das informações disponibilizadas e criação do serviço de informações aos cidadãos (SIC) são evidenciados por 34,15\% dos municípios estudados. O indicador referente às informações quanto aos contratos celebrados é disponibilizado por $36,59 \%$ dos municípios analisados, percentual superior ao apurado pelo TCE-RS (2012) de 18,55\%.

Quanto a indicação clara da LAI nas páginas eletrônicas das prefeituras, apurouse que quase a metade dos municípios estudados, ou seja, $48,78 \%$ das prefeituras disponibilizam tal informação. Este percentual é muito superior ao obtido pelo TCE-RS (2012) que identificou esta informação em apenas 4,03\% dos municípios. A indicação de meios para solicitação de informações através das páginas eletrônicas é disponibilizada por $53,66 \%$ dos municípios da amostra, percentual também muito superior ao obtido pelo TCE-RS (2012) de 4,64\%.

A disponibilidade de uma ferramenta de pesquisa na página eletrônica é evidenciada por $56,10 \%$ dos municípios analisados. O trabalho desenvolvido pelo 
Nível de transparência por meio da evidenciação de informações obrigatórias de municípios do

Rio Grande do Sul

Vagner Naysinger Machado, Simone Beatriz Santos da Silva Marques, Clea Beatriz Macagnan

(TCE-RS, 2012) apontou que 35,08\% dos municípios gaúchos disponibilizavam em suas páginas eletrônicas a ferramenta de pesquisa. Além disso, a evidenciação das informações quanto às competências das secretarias que compõem a gestão municipal é divulgada por $68,29 \%$ das prefeituras estudadas, contra $29,80 \%$ obtidos no estudo realizado pelo TCE-RS (2012).

A Tabela 9 mostra os indicadores previstos na LAI evidenciados por mais de $70 \%$ dos municípios pesquisados.

Tabela 9 - Indicadores da LAI evidenciados por mais de 70\% dos municípios

\begin{tabular}{ccc}
\hline Indicadores (Informações) & № Município & $\%$ \\
\hline Permissão para gravação de relatórios eletrônicos & 41 & 100,00 \\
Informações quanto estrutura organizacional & 39 & 95,12 \\
Informações sobre despesas realizadas & 38 & 92,68 \\
Informações sobre licitações, editais e resultados dos certames & 33 & 80,49 \\
Divulgação do endereço, telefones e horário de atendimento da(s) & 33 & 80,49 \\
unidade(s) & & \\
\hline
\end{tabular}

Fonte: Dados da pesquisa.

Os endereços, telefones e horários de atendimento das prefeituras e informações quanto a licitações, editais e resultados dos certames são disponibilizados nas páginas eletrônicas de trinta e três $(80,49 \%)$ municípios. Informações quanto às despesas realizadas constam em $92,68 \%$ das páginas eletrônicas das prefeituras, o que difere dos $21,98 \%$ obtidos no estudo do TCE-RS (2012). Já as informações quanto à estrutura organizacional das prefeituras são evidenciadas nas páginas eletrônicas de 95,12\% dos municípios pesquisados, o que se assemelha aos $84,68 \%$ obtidos no estudo do TCERS (2012). Por fim, o único indicador evidenciado em todas as páginas dos municípios estudados corresponde à permissão de gravação dos relatórios eletrônicos disponíveis nas páginas eletrônicas das prefeituras. Como o TCE-RS (2012) adotou metodologia diferente da empregada nessa pesquisa, não foi possível efetuar comparação entre os resultados obtidos em ambos os estudos. 
Nível de transparência por meio da evidenciação de informações obrigatórias de municípios do

Rio Grande do Sul

Vagner Naysinger Machado, Simone Beatriz Santos da Silva Marques, Clea Beatriz Macagnan

\section{CONSIDERAÇÕES FINAIS}

A transparência na evidenciação das informações quanto à gestão pública é peça fundamental para o desenvolvimento do efetivo controle social por parte da sociedade. Assim, uma política de transparência que tenha como foco a qualidade e quantidade das informações disponibilizadas fornece aos usuários desta informação, os cidadãos, parâmetros para nortear suas decisões, tanto na escolha de seus representantes como na fiscalização dos seus atos.

A pesquisa realizada nas páginas eletrônicas de 41 municípios do Rio Grande do Sul com mais de 50 mil habitantes, revela que em nenhuma foram evidenciados os 32 indicadores requeridos na LRF e na LAI, ficando o nível de transparência da gestão pública municipal abaixo de $50 \%$, o que pode ser considerado baixo. Esses dados revelam que a Internet, como instrumento de interação entre o Estado e a sociedade, deve ser mais bem aproveitada, no sentido de uma maior evidenciação das informações por parte dos gestores públicos municipais.

Quanto ao cumprimento dos dispositivos legais analisados, observou-se que o atendimento aos dispositivos da Lei de Acesso à Informação é maior que os requeridos na Lei de Responsabilidade Fiscal. Informações relacionadas à participação popular nos processos de elaboração do PPA, LDO e LOA são omitidas por todos os municípios analisados e a maioria não evidencia as informações ligadas a accountability (prestação de contas). Entretanto, todos os municípios pesquisados disponibilizam a gravação dos relatórios eletrônicos em suas páginas eletrônicas e a maioria evidencia informações acerca da estrutura administrativa da prefeitura, assim como informações quanto às despesas realizadas.

Os dados obtidos indicam que as prefeituras dos municípios estudados necessitam desenvolver uma política de transparência mais efetiva, voltada para a evidenciação das informações obrigatórias em suas páginas eletrônicas, visando oportunizar aos cidadãos o desenvolvimento de um efetivo controle social. Nesse sentido, a Convenção das Nações Unidas Contra a Corrupção (ONU, 2003) menciona 
Nível de transparência por meio da evidenciação de informações obrigatórias de municípios do

Rio Grande do Sul

Vagner Naysinger Machado, Simone Beatriz Santos da Silva Marques, Clea Beatriz Macagnan

que uma das medidas preventivas em relação à corrupção é o aumento da transparência na gestão pública, por meio do amplo acesso por parte da sociedade às informações. Logo, ao se apontar que as prefeituras não estão disponibilizando de forma ampla informações sobre os atos de seus gestores, pode-se concluir que há um cenário propicio a práticas ilícitas e ao mau uso dos recursos públicos por parte dos mesmos.

Sugere-se a ampliação da análise a municípios de outros Estados do Brasil, para verificar o nível de acesso às informações por parte dos cidadãos em um contexto nacional. Além disso, aspectos quanto à evidenciação de informações de caráter voluntário, possíveis fatores explicativos dos níveis de transparência apurados e uma análise voltada à qualidade das informações constantes da política de transparência da gestão pública municipal, podem servir de norte a pesquisas futuras.

\section{REFERÊNCIAS}

AGÊNCIA DE NOTÍCIAS DOS DIREITOS DA INFÂNCIA (ANDI). (2013). Acesso à informação e controle social das políticas públicas, artigo 19. Brasília - DF, 2009. Disponível em: < http://www.andi.org.br>. Acesso em: 06/fev.

ARRUDA, M. F; TELES, J. S. (2010). A importância do controle social na fiscalização dos gastos públicos. Revista Razão Contábil e Finanças, Fortaleza, n.1.

AVELINO, B. C; COLAUTO, R. D; CUNHA, J. V. A. (2010). Evidenciação, Teoria da Legitimidade e Hipótese dos Custos Políticos: Estudo no Governo Estadual e Municipal de Minas Gerais. In: CONGRESSO USP DE INICIAÇÃO CIENTÍFICA EM CONTABILIDADE, FIPECAFI, 7, 2010, São Paulo/SP. Anais. São Paulo, SP. CD-ROM.

BHATTA, G. (2003). Post-NPM themes in public sector governance. State Services Commission. n. 17.

BRASIL. Lei Complementar no 101, de 04 de maio de 2000. Institui normas de finanças públicas voltadas para a responsabilidade na gestão fiscal e dá outras providências. Brasília, DF, 04 de maio de 2000. Disponível em: <http://www.planalto.gov.br/ccivil_03/Leis/LCP/Lcp101.htm>. Acesso em: 04/jan/2013. 
Nível de transparência por meio da evidenciação de informações obrigatórias de municípios do

Rio Grande do Sul

Vagner Naysinger Machado, Simone Beatriz Santos da Silva Marques, Clea Beatriz Macagnan

BRASIL, Lei no. 12.527, de 18 de novembro de 2011. Regula o acesso a informação previsto no inciso XXXIII do art. 5ㄴ, no inciso II do $\S 3^{\circ}$ do art. 37 e no $\S 2^{\circ}$ do art. 216 da Constituição Federal; altera a lei oㅡ 8.112, de 11 de dezembro de 1990; revoga a Lei oㅜ 11.111, de 5 de maio de 2005, e dispositivos da Lei oㅡ 8.159, de 8 de janeiro de 1991; e dá outras providências. Brasília, DF, 18 de novembro de 2011. Disponível em:< http://www.planalto.gov.br/ccivil_03/_ato2011-2014/2011/lei/l12527.htm>. Acesso em: 21/dez/2012.

BUSHMAN, R. M; PIOTROSKI, J. D; SMITH, A. J. (2004). What determines corporate transparency? Journal of accounting research, v. 42, n. 2, p-207-253.

CONTROLADORIA-GERAL DA UNIÃO (CGU). Acesso à Informação Pública: Uma Introdução à Lei no 12.527, de 18 de novembro de 2011. Brasília (DF), 2011. Disponível em:

<http://www.acessoainformacao.gov.br/acessoainformacaogov/publicacoes/CartilhaAce ssoalnformacao.pdf>. Acesso em 15/dez/2012.

COSTA, J. F. da. (2012). Reflexos da Lei de Responsabilidade Fiscal no endividamento dos Municípios brasileiros. In: CONGRESSO BRASILEIRO DE CONTABILIDADE CFC, 18, 2008, Gramado, RS. Anais eletrônicos... Brasília/DF, 2008. Disponível em: <http://www.congressocfc.org.br/hotsite/trabalhos_1/334.pdf >. Acesso em 14/dez.

CRUZ, C. F.; FERREIRA, A. C. de S; SILVA, L. M. da; MACEDO, M. Á. da S. Transparency of the municipal public management: a study from the homepages of the large Brazilian municipalities. Rev. Adm. Pública [online]. 2012, vol.46, n.1, pp. 153-176. ISSN 0034-7612. Doi: http://dx.doi.org/10.1590/S0034-76122012000100008.

FERREIRA, C. M. M. (1996). Crise e reforma do Estado: uma questão de cidadania e valorização do servidor. Revista do Serviço Público. v.120, n. 3.

FOX, J. (2007). Government Transparency and policymaking. Public Choice, n.131, p. 23-44.

GERIGK, W. (2008). O impacto da Lei de Responsabilidade Fiscal sobre a Gestão Financeira dos pequenos municípios do Paraná. 2008. 315f. (Dissertação de Mestrado). Programa de Mestrado em Contabilidade, Setor de Ciências Sociais Aplicadas. Universidade Federal do Paraná, Curitiba, PR.

GUPTA, A. (2008). Transparency Under Scrutiny: Information Disclosure in Global Environmental Governance. Global environmental politics. v. 8, n. 2.

INSTITUTO BRASILEIRO DE GEOGRAFIA E ESTATísTICA. Contagem da População 2010. em: 
Nível de transparência por meio da evidenciação de informações obrigatórias de municípios do

Rio Grande do Sul

Vagner Naysinger Machado, Simone Beatriz Santos da Silva Marques, Clea Beatriz Macagnan

http://www.ibge.gov.br/home/estatistica/populacao/censo2010/default.shtm >. Acesso em: 14/dez/2012.

KRISTIANSEN, S; PRAMUSINTO, A. D.A; PUTRANTO, E.A. (2008). Public Sector reforms and Financial Transpancy: Experiences from Indonesian Districts. Contemporary Southeast Asia. v. 21, n.1, p. 64-87.

LOCK, F. do N. (2003). Transparência da gestão municipal através das informações contábeis divulgadas na internet. 2003. 111 f. (Dissertação de Mestrado). Universidade Federal de Pernambuco, Recife.

ORGANIZAÇÃO DAS NAÇÕES UNIDAS (ONU). (2003). Convenção das Nações Unidas contra a corrupção. Disponível em:< http://www.planalto.gov.br/ccivil_03/_Ato2004-2006/2006/Decreto/D5687.htm>. Acesso em: 01/fev/2013.

RELLY, J. E; SABHARWAL, M. (2009). Perceptions of transparency of government policymaking: A cross-national study. Government Information quarterly, v.26, p.148157.

SACRAMENTO, A. R. S; PINHO, J. A. G. (2007). Transparência na administração pública: o que mudou depois da lei de responsabilidade fiscal: um estudo exploratório em seis municípios da região metropolitana de Salvador. Salvador, BA (Brasil). Revista de Contabilidade da UFBA, v. 1, n. 1.

SANTANA JUNIOR, J. J. B. (2008). Transparência fiscal eletrônica: uma análise dos níveis de transparência apresentados nos sites dos poderes e órgãos dos estados e do Distrito Federal do Brasil. 177 f. (Dissertação de Mestrado). Programa Multinstitucional e Inter-regional de Pós-Graduação em Ciências Contábeis, Universidade de Brasília, Universidade Federal de Pernambuco, Universidade Federal da Paraíba e Universidade Federal do Rio Grande do Norte, Recife.

SILVA, L. M. (2009). Contabilidade governamental: um enfoque administrativo. (8 ed.). São Paulo: Atlas.

SOUZA, A. A. de; BOINA, T. M; AVELAR, E. A; GOMIDE, P. L. R. (2008). Evidenciação Contábil nos Municípios Mineiros: Atendimento ao artigo 48 da Lei de Responsabilidade Fiscal. In: CONGRESSO BRASILEIRO DE CONTABILIDADE CFC, 18. Gramado, RS. Anais eletrônicos... Brasília, em:<http://congressocfc.org.br/hotsite/trabalhos_1/486.pdf>. Acesso em: 20/dez/2012. 
Nível de transparência por meio da evidenciação de informações obrigatórias de municípios do

Rio Grande do Sul

Vagner Naysinger Machado, Simone Beatriz Santos da Silva Marques, Clea Beatriz Macagnan

STYLES, A. K; TENNYSON, M. (2007). The accessibility of financial reporting of U.S. Municipalities on the Internet. Journal of Public Budgeting, accounting \& financial Management. V. 19, n.1, p. 56-92.

TOLENTINO, M. A; FEITOSA, M. N; SANTIAGO, W. P; DUARTE, S. V. (2002). Lei de Responsabilidade Fiscal: Dificuldades e Benefícios da Implementação e Operacionalização na Microrregião de Bocaiúva. Unimontes Científica. Montes Claros, (MG), v.3, n.3.

TRIBUNAL DE CONTAS DO ESTADO DO RIO GRANDE DO SUL. Os Portais dos Municípios Gaúchos (Poder Executivo) Análise em face da lei Federal no 12.527/2011 (Lei de Acesso à informação). Disponível em:< https://portal.tce.rs.gov.br/portal/page/portal/noticias_internet/textos_diversos_pente_fin o/Relatorio_LAI_Executivo_Municipal.pdf>. Acesso em: 22/nov/2012.

Data de Submissão: 28/09/2013 Data de Aceite: 18/05/2014 